\title{
Noiseless manipulation of helical edge state transport by a quantum magnet
}

\author{
P. G. Silvestrov, ${ }^{1}$ P. Recher, ${ }^{1,2}$ and P. W. Brouwer ${ }^{3}$ \\ ${ }^{1}$ Institute for Mathematical Physics, TU Braunschweig, 38106 Braunschweig, Germany \\ ${ }^{2}$ Laboratory for Emerging Nanometrology Braunschweig, 38106 Braunschweig, Germany \\ ${ }^{3}$ Physics Department and Dahlem Center for Complex Quantum Systems, Freie Universität Berlin, Arnimallee 14, 14195 Berlin, Germany
}

(Received 10 November 2015; revised manuscript received 19 April 2016; published 16 May 2016)

\begin{abstract}
The current through a helical edge state of a quantum spin Hall insulator may be fully transmitted through a magnetically gapped region due to a combination of spin-transfer torque and spin pumping [Meng et al., Phys. Rev. B 90, 205403 (2014)]. Using a scattering approach, we here argue that in such a system the current is effectively carried by electrons with energies below the magnet-induced gap and well below the Fermi energy. This has striking consequences, such as the absence of shot noise, an exponential suppression of thermal noise, and an obstruction of thermal transport. For two helical edges covered by the same quantum magnet, the device can act as a robust noiseless current splitter.
\end{abstract}

DOI: 10.1103/PhysRevB.93.205130

A time-reversal symmetry breaking magnetic field is well known to introduce backscattering of helical edge states and to destroy the conductance quantization of a quantum spin Hall insulator [1-4]. More subtle is the case of the effective magnetic field created by dynamic spinful impurities [5-9]. The reason for that is that a spin flip is necessary to reflect an electron in a helical edge state. In the case of a small impurity spin the magnetic impurities immediately become fully polarized, leaving no room for anymore backscattering of the current $[5,6]$.

At first sight, the situation is different for a helical edge coupled to a magnet, because for a magnet the backscattering of a single electron in the helical edge happens without a complete change of the magnet's macroscopic spin polarization. Moreover, the exchange coupling to a magnet opens up a gap in the helical-state spectrum, similar to the gap opened by a magnetic field. Yet, as was shown recently by Meng et al. [9], under certain conditions concerning the magnet's anisotropy energy, an electrical current incident on the magnet is fully transmitted. Reference [9] invokes a combination of spin-transfer torque and spin pumping [10-15] as the cause of this effect. The system considered in Ref. [9] was recently suggested as an "adiabatic quantum motor" [16].

In this paper we show that such a macroscopic magnet coupled to the helical edge of a quantum spin Hall insulator has very special noise and thermal transport properties, some of which are unparalleled in the field of mesoscopic quantum transport: Thermal transport and shot noise are essentially absent in a two-terminal setup, and a multiterminal geometry involving a magnet coupled to two helical edges allows a current partitioning without shot noise. The origin of these remarkable phenomena is that all electrons close to the chemical potential $\mu$ are reflected if $\mu$ is inside the magnetinduced gap, whereas the current is effectively carried by electrons with energy below the gap, which may be very large compared to both temperature and applied bias-a situation reminiscent of the interrelation of electrons at low and high energy in the case of the chiral anomaly [17].

We investigate the same system as in Ref. [9], including the same conditions on the magnet's anisotropy energy (see below), but we use the scattering approach $[18,19]$ to describe transport. In addition to rederiving the results of Ref. [9] in a more general context, not being restricted to a magnet that fully gaps the helical edge state, the scattering approach gives us a unified framework for the description of charge and energy transport and noise-related phenomena. Reflection of helical electrons off the magnet is inelastic, changing both their spin and energy when a bias voltage is applied, the sign of the energy change depending on whether the electron is incident from the source or the drain. Together, transmitted electrons with energies below the magnet-induced gap and reflected electrons in the helical edge form a noiseless current-carrying state.

Model and scattering matrix. The interaction of the helical edge state with the magnet is described by the secondquantized Hamiltonian [9]

$$
H=\int d x \hat{\boldsymbol{\psi}}_{\mathbf{\mathrm { x }}}^{\dagger}\left[-i \hbar v_{\mathrm{F}} \partial_{x} \sigma_{z}+h(x) \boldsymbol{\sigma} \cdot \mathbf{M}\right] \hat{\boldsymbol{\psi}}_{\mathbf{x}}+\frac{D}{2} M_{z}^{2}
$$

Here $v_{\mathrm{F}}$ is the Fermi velocity, $\sigma_{x, y, z}$ are the Pauli matrices acting on the spinor $\hat{\psi}_{\mathbf{x}}=\left[\hat{\psi}_{\uparrow}(x), \hat{\psi}_{\downarrow}(x)\right]^{\mathrm{T}}$ of the helical edge states, and $h(x)$ is a function that describes the exchange coupling between the magnetic moment $\mathbf{M}$ and the edge state spin (both measured in units of $\hbar$ ), such that $h(x)=0$ for $x \rightarrow \pm \infty$. A schematic picture of the arrangement is shown in Fig. 1(a).

We employ a macrospin approximation, i.e., the magnetic moment $\mathbf{M}$ in Eq. (1) is the only collective variable describing the dynamics of the macroscopic magnet. The last term in Eq. (1) represents the magnetic anisotropy energy. We take $D>0$, corresponding to easy-plane anisotropy. The Hamiltonian (1) is invariant under spin rotations in the $x-y$ plane, so that the $z$ component of the total spin $M_{z}+\sigma_{z}$ is conserved. This additional symmetry of the model is the key to the absence of backscattering in the steady state (in the absence of residual interaction effects inside the edge) $[5,6,9]$. We first derive this result for the mean current, and then extend our discussion to noise and distribution functions.

Prior to considering the full many-body case it is convenient to start with a single electron problem in the topologicalinsulator edge interacting with the magnetic moment, described by the first-quantized Hamiltonian

$$
H=-i \hbar v_{\mathrm{F}} \partial_{x} \sigma_{z}+h(x) \boldsymbol{\sigma} \cdot \mathbf{M}+\frac{1}{2} D M_{z}^{2} .
$$


(a)

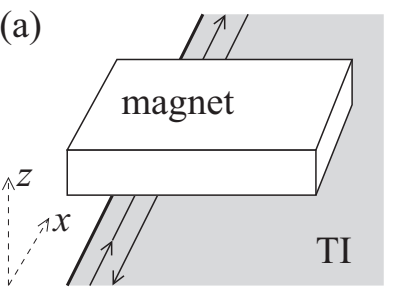

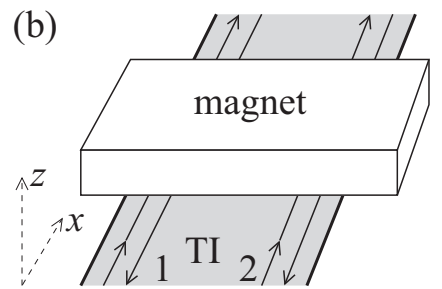

(b)

FIG. 1. Schematic drawing of the geometry we consider: One helical edge (a) or both edges (b) of a two-dimensional topological insulator (gray) exchange coupled to a magnetic insulator.

A reflection of an electron at the topological-insulator edge is accompanied by a unit change $M_{z} \rightarrow M_{z} \pm 1$ of the $z$ component of the magnetization, where the + and - signs refer to electrons incident from the left or from the right, respectively. The Hamiltonian (2) thus decouples into sectors in which the magnetization has the value $M_{z}$ for right-moving electrons and $M_{z}+1$ for left-moving electrons. Inside such a sector one may obtain a pure scattering problem by performing the unitary transformation (analogous to the transformation to the rotating frame of Refs. $[9,15])$

$$
\tilde{H}=\left(\begin{array}{cc}
1 & 0 \\
0 & m_{-}
\end{array}\right) H\left(\begin{array}{cc}
1 & 0 \\
0 & m_{+}
\end{array}\right),
$$

where $\quad m_{+}=\left(M_{x}+i M_{y}\right) / M_{\perp}, \quad$ with $\quad M_{\perp}=$ $\sqrt{\left(M-M_{z}\right)\left(M+1+M_{z}\right)}$, is the operator that raises the value of $M_{z}$ by unity. Using the equality $m_{-} m_{+}=1$ and omitting constant terms, we have (cf. [9])

$$
\tilde{H}=\left[-i \hbar v_{\mathrm{F}} \partial_{x}+h(x) M_{z}-\frac{\hbar \omega}{2}\right] \sigma_{3}+M_{\perp} h(x) \sigma_{1},
$$

with $\hbar \omega=D\left(M_{z}+1 / 2\right)$ the difference of the anisotropy energies between the states with magnetization $M_{z}$ and $M_{z}+1$. In Eq. (4) we neglected small terms $\sim h$ in comparison to the large term $\sim h M_{z}$. In the Hamiltonian $\tilde{H}$ the $z$ component of the magnetization can be considered constant. The reflection and transmission amplitudes $r(\varepsilon), r^{\prime}(\varepsilon), t(\varepsilon)$, and $t^{\prime}(\varepsilon)$ for the scattering problem (4) at energy $\varepsilon$ can then be found using standard methods. (Primed amplitudes are for electrons incident from the right.) In particular, for a smooth function $h(x)$ having a wide maximum the Hamiltonian has a gapped region with maximal gap $2 \varepsilon_{\text {gap }}$, with $\varepsilon_{\text {gap }}=\max _{x} h(x) M_{\perp}$. For our considerations it will be important that $r(\varepsilon) \rightarrow 0$ if $|\varepsilon| \gg \varepsilon_{\text {gap }}$.

The frequency $\omega$ appearing in Eq. (4) is the frequency of rotation of the classical magnetization $\mathbf{M}$ around the $z$ axis. Note that outside the magnet region, the kinetic energies of the left-moving and the right-moving electrons are $\varepsilon_{-}=$ $\varepsilon-\hbar \omega / 2$ and $\varepsilon_{+}=\varepsilon+\hbar \omega / 2$, respectively. Considering the kinetic energies separately is important, because they appear in the distribution function of incoming electrons [see Eq. (7) below].

Transforming back to the original formulation, Eq. (2), the scattering problem can be written in second-quantized form as

$$
\begin{aligned}
& \hat{b}_{\mathrm{L}}\left(\varepsilon_{-}\right)=r(\varepsilon) m_{+} \hat{a}_{\mathrm{L}}\left(\varepsilon_{+}\right)+t^{\prime}(\varepsilon) \hat{a}_{\mathrm{R}}\left(\varepsilon_{-}\right), \\
& \hat{b}_{\mathrm{R}}\left(\varepsilon_{+}\right)=r^{\prime}(\varepsilon) m_{-} \hat{a}_{\mathrm{R}}\left(\varepsilon_{-}\right)+t(\varepsilon) \hat{a}_{\mathrm{L}}\left(\varepsilon_{+}\right),
\end{aligned}
$$

where the operators $\hat{b}_{\mathrm{L}}(\varepsilon), \hat{b}_{\mathrm{R}}(\varepsilon)$ and $\hat{a}_{\mathrm{L}}(\varepsilon), \hat{a}_{\mathrm{R}}(\varepsilon)$ annihilate an outgoing and an incomingelectron [19] at a kinetic energy $\varepsilon$, respectively, at the left $(\mathrm{L})$ and the right $(\mathrm{R})$ of the magnet.

So far we have considered the problem of a single electron scattering off the magnetic moment $\mathbf{M}$. The scattering amplitudes $r(\varepsilon), r^{\prime}(\varepsilon), t(\varepsilon)$, and $t^{\prime}(\varepsilon)$, as well as the energy shift $\hbar \omega$ are functions of $M_{z}$. When considering the many-particle problem, in principle, $M_{z}$ is a fluctuating quantity, because of the simultaneous scattering off the magnetic moment of multiple electrons. However, in the limit of a macroscopic magnetic moment $M$ relative fluctuations of the out-ofplane magnetization $M_{z}$ are small and one may evaluate the amplitudes $r(\varepsilon), r^{\prime}(\varepsilon), t(\varepsilon)$, and $t^{\prime}(\varepsilon)$, as well as the energy shift $\hbar \omega$ at the mean value $\left\langle M_{z}\right\rangle$. With this approximation, Eq. (5) can be applied to the many-particle system. Employing the eigenfunctions of Eq. (4) in this mean-field way is equivalent to the approach of Ref. [9].

Current. The charge current through the helical edge is calculated using the expression [19]

$$
I_{\mathrm{L}}=\frac{e}{h} \int d \varepsilon d \varepsilon^{\prime}\left[a_{\mathrm{L}}^{\dagger}(\varepsilon) a_{\mathrm{L}}\left(\varepsilon^{\prime}\right)-b_{\mathrm{L}}^{\dagger}(\varepsilon) b_{\mathrm{L}}\left(\varepsilon^{\prime}\right)\right]
$$

for the current to the left of the magnet, and a similar expression for the current $I_{\mathrm{R}}$ to the right of the magnet. For the incoming states one has

$$
\left\langle a_{\alpha}^{\dagger}(\varepsilon) a_{\beta}\left(\varepsilon^{\prime}\right)\right\rangle=f_{\alpha}(\varepsilon) \delta\left(\varepsilon-\varepsilon^{\prime}\right) \delta_{\alpha \beta}, \alpha, \beta=\mathrm{L}, \mathrm{R},
$$

where $f_{\alpha}(\varepsilon)=1 /\left[e^{\left(\varepsilon-\mu_{\alpha}\right) / k_{\mathrm{B}} T_{\alpha}}+1\right]$ is the distribution function for reservoir $\alpha$, with chemical potential $\mu_{\alpha}$ and temperature $T_{\alpha}, \alpha=$ L, R. Substituting Eq. (5) and using $|t(\varepsilon)|^{2}=\left|t^{\prime}(\varepsilon)\right|^{2}$, we find

$$
I_{\mathrm{L}}=\frac{e}{h} \int d \varepsilon\left[f_{\mathrm{L}}\left(\varepsilon_{-}\right)-|r(\varepsilon)|^{2} f_{\mathrm{L}}\left(\varepsilon_{+}\right)-|t(\varepsilon)|^{2} f_{\mathrm{R}}\left(\varepsilon_{-}\right)\right] .
$$

When a bias voltage $e V$ is applied across the magnet,

$$
\mu_{\mathrm{L}}=\mu+e V / 2, \mu_{\mathrm{R}}=\mu-e V / 2,
$$

initially the reflections of electrons incident from the left and from the right will not be in balance. Since each reflection leads to a change $\Delta M_{z}= \pm 1$, with a + sign for electrons incident from the left and a - sign for electrons incident from the right, the application of a bias leads to a finite out-of-plane magnetization component $M_{z}$. The rate of change of $M_{z}$ is the difference of reflection rates for electrons incident from the left and from the right,

$$
\left\langle\dot{M}_{z}\right\rangle=\frac{1}{h} \int d \varepsilon|r(\varepsilon)|^{2}\left[f_{\mathrm{L}}\left(\varepsilon_{+}\right)-f_{\mathrm{R}}\left(\varepsilon_{-}\right)\right] .
$$

Note that the integral is convergent, because $r(\varepsilon) \rightarrow 0$ for $|\varepsilon| \gg \varepsilon_{\text {gap }}$. If the two contacts are held at the same temperature, the integrand in Eq. (10) never changes sign, and the stationary condition $\left\langle\dot{M}_{z}\right\rangle=0$ may be achieved at $\hbar \omega=e V$ only, i.e., when the integrand vanishes at all $\varepsilon$. The substitution of Eq. (10) into Eq. (8) then yields the stationary current (using $\left.|r(\varepsilon)|^{2}+|t(\varepsilon)|^{2}=1\right)$

$$
I_{\mathrm{R}}=I_{\mathrm{L}}=\frac{e}{h} \int d \varepsilon\left[f_{\mathrm{L}}\left(\varepsilon_{-}\right)-f_{\mathrm{R}}\left(\varepsilon_{-}\right)\right]=\frac{e^{2}}{h} V .
$$

This is the reflectionless current originally obtained by Meng et al. [9], who arrive at the equality $e V=\hbar \omega$ as a direct 


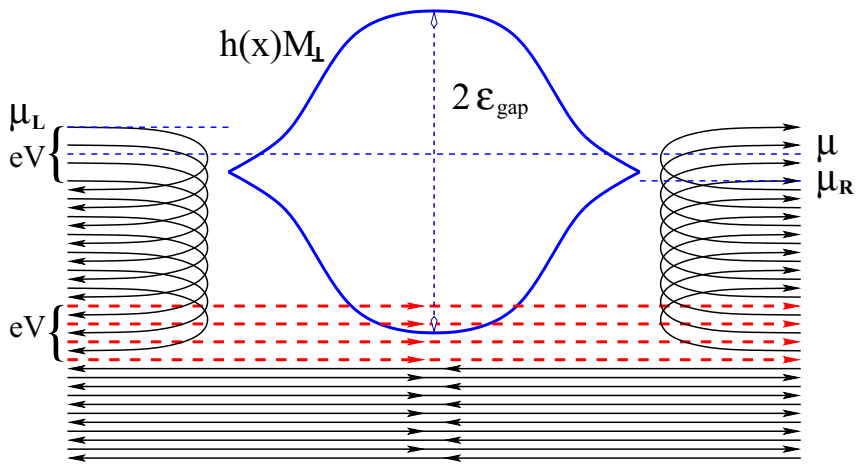

FIG. 2. Schematic illustration of reflection and transmission as a function of energy, for the special case that the exchange coupling $h(x)$ is a smooth function of $x$ and the chemical potential $\mu$ lies inside the spectral gap induced by the coupling to the ferromagnet. The vertical axis refers to the electrons' kinetic energy, which changes by an amount $\hbar \omega=e V$ upon reflection. Electrons carrying the actual current are shown by red dashed lines. Although details change if the coupling function $h(x)$ is not smooth, the conclusion that the current is carried effectively by electrons far away from the Fermi level remains true as long as the chemical potential is inside the gap [20].

consequence of the transformation to a frame corotating with the precessing magnetization.

In Fig. 2 we schematically illustrate which electrons carry the current in the special case that the function $h(x)$ is smooth, so that the reflection probability $|r(\varepsilon)|^{2}=\Theta\left(\varepsilon_{\text {gap }}-|\varepsilon|\right), \Theta$ being the step function, and with chemical potential $\mu$ in the gap [20]. Since the kinetic energy for electrons incoming from the left reservoir is $\varepsilon_{+}=\varepsilon+\hbar \omega / 2$, electrons incident from the left are fully reflected if (and only if) their kinetic energy $-\varepsilon_{\text {gap }}+\hbar \omega / 2<\varepsilon_{+}<\varepsilon_{\text {gap }}+\hbar \omega / 2$ (i.e., $-\varepsilon_{\text {gap }}<\varepsilon<\varepsilon_{\text {gap }}$, although we stress that it is the kinetic energy $\varepsilon_{ \pm}=\varepsilon \pm \hbar \omega / 2$ that matters for the distribution functions). Similarly, electrons incident from the right are fully reflected if and only if their kinetic energy $\varepsilon_{-}=\varepsilon-\hbar \omega / 2$ is between $-\varepsilon_{\text {gap }}-\hbar \omega / 2$ and $\varepsilon_{\text {gap }}-\hbar \omega / 2$. Hence, the current is carried effectively by electrons incident from the left, with kinetic energies between $-\varepsilon_{\text {gap }}-\hbar \omega / 2$ and $-\varepsilon_{\text {gap }}+\hbar \omega / 2$. These are electrons far below the Fermi level, as shown in the figure.

Note that, while the qualitative picture described by Fig. 2 relies on the chemical potential $\mu$ being inside the gap and on the function $h(x)$ being smooth (such that the electrons with energies outside the gap are fully transmitted), the conclusion (11) of a perfectly transmitted current does not rely on these conditions. The only change in the case of a chemical potential outside the gap is an increased relaxation time because of the small reflection coefficient $r(\varepsilon)$ for energies near $\mu$ [see Eq. (10)]. The reflection probability can be increased and the relaxation time lowered by making the edge-to-magnet coupling nonadiabatic, e.g., by engineering the function $h(x)$ with sharp features.

Noise. Since Eq. (11) predicts a perfect transmission of the current, one should expect no zero-frequency shot noise. This follows directly for the special case considered in Fig. 2, where all the right-moving states with energies below $\mu_{\mathrm{L}}$ and all the left-moving states with energies below $\mu_{\mathrm{R}}$ are occupied, leaving no room for any uncertainty, i.e., for noise. Figure 2 also suggests a strong suppression of the thermal noise in the case of the chemical potential inside the gap, since in that case all electrons with energy near the chemical potential-i.e., all electrons that "know" about the temperature-are reflected.

To formally calculate the noise we may use the scattering matrix (5). The zero-frequency noise power then takes the form (cf. Ref. [19])

$$
\begin{aligned}
S= & \frac{2 e^{2}}{h} \int d \varepsilon\left(| t ( \varepsilon ) | ^ { 2 } \left\{f_{\mathrm{L}}\left(\varepsilon_{+}\right)\left[1-f_{\mathrm{L}}\left(\varepsilon_{+}\right)\right]\right.\right. \\
& \left.+f_{\mathrm{R}}\left(\varepsilon_{-}\right)\left[1-f_{\mathrm{R}}\left(\varepsilon_{-}\right)\right]\right\} \\
& \left.+|t(\varepsilon)|^{2}\left[1-|t(\varepsilon)|^{2}\right]\left[f_{\mathrm{L}}\left(\varepsilon_{+}\right)-f_{\mathrm{R}}\left(\varepsilon_{-}\right)\right]^{2}\right) .
\end{aligned}
$$

For equal lead temperatures $T=T_{\mathrm{R}}=T_{\mathrm{L}}$ and in the stationary limit $\hbar \omega=e V$ one has $f_{\mathrm{R}}\left(\varepsilon_{-}\right)=f_{\mathrm{L}}\left(\varepsilon_{+}\right)$[see Eq. (10)], so that the last line in Eq. (12), the shot noise, vanishes. What remains is the thermal noise, which upon using the equality $f_{\mathrm{R}}\left(\varepsilon_{-}\right)=f_{\mathrm{L}}\left(\varepsilon_{+}\right)$, becomes

$$
S=\frac{4 e^{2}}{h} \int d \varepsilon|t(\varepsilon)|^{2} f_{\mathrm{L}}\left(\varepsilon_{+}\right)\left[1-f_{\mathrm{L}}\left(\varepsilon_{+}\right)\right] .
$$

The thermal noise depends in general on the specific form of $t(\varepsilon)$. For a smooth $h(x)$ one has $|t(\varepsilon)|^{2}=\Theta\left(|\varepsilon|-\varepsilon_{\text {gap }}\right)$, the energy integration is easily done, and one finds

$$
S=\frac{8 e^{2} k_{\mathrm{B}} T}{h} e^{-\varepsilon_{\text {gap }} / k_{\mathrm{B}} T} \cosh \left(\mu / k_{\mathrm{B}} T\right),
$$

plus corrections that vanish in the limit $\left|\varepsilon_{\text {gap }} \pm \mu\right| \gg k_{\mathrm{B}} T$. If the function $h(x)$ is not smooth the detailed expression for the shot noise power changes, but not the conclusion that $S$ is exponentially small in $\min \left(\varepsilon_{\text {gap }} \pm \mu\right)$. Clearly the exponential suppression of the thermal noise indicates a departure from the usual form $S_{\mathrm{Th}}=4 k_{\mathrm{B}} T d I / d V$ for two-terminal conductors [19]. For chemical potential $\mu$ well outside the gap region the thermal noise obtained from Eq. (13) agrees with the conventional result $S=S_{\mathrm{Th}}$.

Distribution functions. The strong suppression of thermal noise can also be illustrated through a calculation of the distribution functions $f_{\mathrm{R} \text {,out }}$ and $f_{\mathrm{L} \text {,out }}$ for electrons, which have been reflected from or transmitted through the device. One finds

$$
\begin{aligned}
& f_{\mathrm{R}, \text { out }}(\varepsilon)=f_{\mathrm{L}}(\varepsilon)\left|t\left(\varepsilon_{-}\right)\right|^{2}+f_{\mathrm{R}}(\varepsilon-\hbar \omega)\left|r\left(\varepsilon_{-}\right)\right|^{2}, \\
& f_{\mathrm{L}, \text { out }}(\varepsilon)=f_{\mathrm{L}}(\varepsilon+\hbar \omega)\left|r\left(\varepsilon_{+}\right)\right|^{2}+f_{\mathrm{R}}(\varepsilon)\left|t\left(\varepsilon_{+}\right)\right|^{2},
\end{aligned}
$$

where $f_{\mathrm{R}}$ and $f_{\mathrm{L}}$ are the distribution functions of electrons incident from the right and left reservoirs, respectively. For equal reservoir temperatures and in the stationary limit $\hbar \omega=$ $e V$, such that $f_{\mathrm{L}}(\varepsilon)=f_{\mathrm{R}}(\varepsilon-\hbar \omega)$, these equations reduce to $f_{\mathrm{R} \text {, out }}(\varepsilon)=f_{\mathrm{L}}(\varepsilon)$ and $f_{\mathrm{L} \text {, out }}(\varepsilon)=f_{\mathrm{R}}(\varepsilon)$ in accordance with Eq. (11). However, the distributions become nontrivial if the temperatures $T_{\mathrm{L}}$ and $T_{\mathrm{R}}$ are different. If the chemical potential $\mu$ is inside the gap, all electrons with energy close to $\mu$ are reflected, and one finds $f_{\mathrm{R} \text {, out }}(\varepsilon)=f_{\mathrm{R}}(\varepsilon-\hbar \omega), f_{\mathrm{L} \text {, out }}(\varepsilon)=$ $f_{\mathrm{L}}(\varepsilon-\hbar \omega)$, i.e., the device transmits the charge of the incident electrons, but not their "temperature"- the device is a thermal insulator. An example for generic reflection and transmission amplitudes is shown in Fig. 3.

Noiseless partitioning of the current. It is instructive to also consider a four-terminal quantum spin Hall device with two 

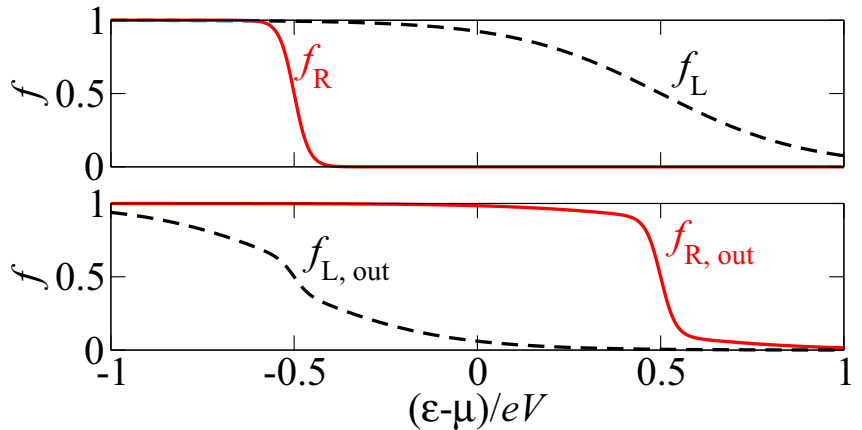

FIG. 3. Illustration of distribution functions for incoming (top) and outgoing (bottom) electrons, for the case $T_{\mathrm{L}} / \mathrm{eV}=0.2, T_{\mathrm{R}} / \mathrm{eV}=$ $0.02,|r(\varepsilon)|^{2}=1-|t(\varepsilon)|^{2}=0.8$. The location of the "step" in the distribution function for the outgoing electrons corresponds to that of the opposite reservoir, consistent with the perfect transmission of the incident current. For this example, the width of the step is, however, predominantly that of the same-side reservoir, consistent with the fact that the device acts as a "thermal insulator."

helical edges covered by the same magnet, as in Fig. 1(b). Let the left contact of the edge 1 be biased by a voltage $V$ and all other terminals be put to ground. The electrons incident from the left contact of edge 1 initiate a precessing nonequilibrium out-of-plane magnetization $M_{z}$, which in turn drives spin and charge currents in the remaining three terminals. An illustrative explanation of the processes taking place in the four-terminal setup is presented in Fig. 4 (similar to Fig. 2 in the two-terminal case). For the explicit calculation the scattering approach used above can be carried over straightforwardly for each edge, as there is no scattering between them. The precession frequency $\omega$ at which a steady state sets in now is given by [20]

$$
\begin{aligned}
0=\left\langle\dot{M}_{z}\right\rangle= & \frac{1}{h} \int d \varepsilon\left\{\left|r_{1}(\varepsilon)\right|^{2}\left[f_{1 \mathrm{~L}}\left(\varepsilon_{+}\right)-f_{1 \mathrm{R}}\left(\varepsilon_{-}\right)\right]\right. \\
& \left.-\left|r_{2}(\varepsilon)\right|^{2}\left[f_{2 \mathrm{~L}}\left(\varepsilon_{-}\right)-f_{2 \mathrm{R}}\left(\varepsilon_{+}\right)\right]\right\},
\end{aligned}
$$

where the labels 1 and 2 refer to the two edges and we have used that the helicity of the edge states is opposite in the two edges. The ability of the magnet to create/change current in each edge state is determined by the reflection coefficient leading to different currents $I_{1}$ and $I_{2}$ for arbitrary $r_{1,2}(\varepsilon)$. However, in the case of all chemical potentials inside the magnet-induced gap with exponential accuracy one has $\left|r_{1}(\varepsilon)\right|=\left|r_{2}(\varepsilon)\right|=$ 1 , leading to $I_{1}=-I_{2}=e^{2} V / 2 h$, independent (again with exponential accuracy) of the possibly different temperatures of the four contacts.

To calculate the noise power one may use Eq. (12) for each edge separately. In the example that all chemical potentials are inside the gap, the currents are obviously noiseless, up to exponentially small corrections in $\left|\varepsilon_{\text {gap } i} \pm \mu_{i}\right| / k_{\mathrm{B}} T$, where $\mu_{i}=\left(\mu_{i \mathrm{~L}}+\mu_{i \mathrm{R}}\right) / 2$ is the average chemical potential in the edge $i$ and $\varepsilon_{\text {gap } i}$ is the value of the gap in the same edge-a result that can already be understood by arguing that in both helical edges the current in Fig. 4 is carried by electrons far below the chemical potential. The absence of noise may be considered surprising, since, unlike in the two-terminal setup, in the four-terminal setup the original incident current appears to be partitioned.

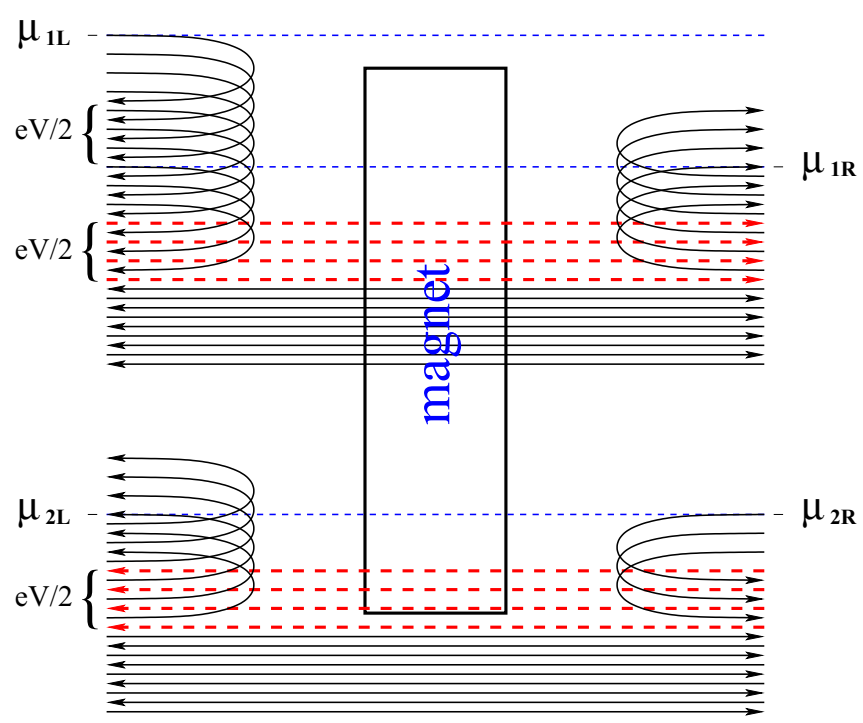

FIG. 4. Noiseless partitioning of the current between the two edges of the topological insulator (TI) covered by the same magnet. The current is injected through the left contact of the upper edge 1 with chemical potential $\mu_{1 \mathrm{~L}}=\mu_{1 \mathrm{R}}+e V$. In the steady state the magnetic moment precesses at a frequency $\hbar \omega=e V / 2$. In this case half of the injected current is reflected back with the energies $\mu_{1 \mathrm{R}}+e V / 2>\varepsilon>\mu_{1 \mathrm{R}}$. In the upper right contact uncompensated right movers have energies $\mu_{1 \mathrm{R}}+e V / 2>\varepsilon>\mu_{1 \mathrm{R}}$. Even though half of the current is reflected, there is no shot noise, since every single electron state is always either fully occupied, or fully empty. Electrons which are physically transmitted from left to right in edge 1 (shown in red) have energies well below the Fermi energy, $\varepsilon \approx-\varepsilon_{1 \text { gap }}$ (cf. Fig. 2). Electrons from the lower edge 2 also have to change their spin and energy upon reflection. This results in the noiseless current $I_{1}=e^{2} V / 2 h=-I_{2}$.

Conclusions. We considered the transport of helical edge state electrons in proximity to a magnet with easy-plane anisotropy compatible with the spin helicity of the edge state. While it was known that (after transient effects) such a system perfectly transmits an incident charge current [9], in spite of the fact that the coupling to the magnetic insulator opens a gap in the spectrum of the helical edge, we have shown that the device has very special noise properties: The current is noiseless, and thermal transport is blocked. We explain this combination of "perfect metal" and "perfect thermal insulator" properties in a single-particle scattering picture, in which effectively the current is carried by electrons with energy far below the chemical potential. In a four-terminal setup, the same device can be used as a noiseless current splitting device.

The authors of Ref. [9] suggest using a mesoscopic $\mathrm{K}_{2} \mathrm{CuF}_{4}$ easy-plane magnetic film for realization of their device. The first experiments with two-dimensional topological insulators used sample widths as small as $\lesssim 1 \mu \mathrm{m}$ [3], which is comparable to the typical size $\gtrsim 1 \mu \mathrm{m}$ of magnetic domains, including those in $\mathrm{K}_{2} \mathrm{CuF}_{4}$ [21]. For that reason, we believe that the same material combination can also be used, in principle, for the four-terminal device of Fig. 1(b).

Our predictions rely strongly on the precise orientation of the magnet's easy-plane anisotropy. While the orientation 
chosen here is generic for a thin magnetic film [22] exchange coupled to the spin polarized helical edge modes [23] of a quantum spin Hall material, small deviations from the ideal limit may still exist. These will be investigated in future work.
Acknowledgments. We acknowledge discussions with B. Probst. This work was supported by the DFG Grant No. RE 2978/1-1 and by the SFB 658 "Elementary Processes in Molecular Switches on Surfaces.”
[1] C. L. Kane and E. J. Mele, Phys. Rev. Lett. 95, 226801 (2005).

[2] B. A. Bernevig, T. L. Hughes, and S. C. Zhang, Science 314, 1757 (2006).

[3] M. König, S. Wiedmann, C. Brüne, A. Roth, H. Buhmann, L. W. Molenkamp, X.-L. Qi, and S.-C. Zhang, Science 318, 766 (2007).

[4] L. Du, I. Knez, G. Sullivan, and R. R. Du, Phys. Rev. Lett. 114, 096802 (2015).

[5] J. Maciejko, C. Liu, Y. Oreg, X.-L. Qi, C. Wu, and S.-C. Zhang, Phys. Rev. Lett. 102, 256803 (2009).

[6] Y. Tanaka, A. Furusaki, and K. A. Matveev, Phys. Rev. Lett. 106, 236402 (2011)

[7] B. L. Altshuler, I. L. Aleiner, and V. I. Yudson, Phys. Rev. Lett. 111, 086401 (2013).

[8] O. M. Yevtushenko, A. Wugalter, V. I. Yudson, and B. L. Altshuler, Europhys. Lett. 112, 57003 (2015).

[9] Q. Meng, S. Vishveshwara, and T. L. Hughes, Phys. Rev. B 90, 205403 (2014).

[10] J. C. Slonczewski, J. Magn. Magn. Mater. 159, L1 (1996).

[11] L. Berger, Phys. Rev. B 54, 9353 (1996).

[12] Y. Tserkovnyak, A. Brataas, and G. E. W. Bauer, Phys. Rev. Lett. 88, 117601 (2002).

[13] Y. Tserkovnyak, A. Brataas, G. E. W. Bauer, and B. I. Halperin, Rev. Mod. Phys. 77, 1375 (2005).

[14] X.-L. Qi, T. L. Hughes, and S.-C. Zhang, Nat. Phys. 4, 273 (2008).
[15] F. Mahfouzi, B. K. Nikolic, S.-H. Chen, and C.-R. Chang, Phys. Rev. B 82, 195440 (2010)

[16] L. Arrachea and F. von Oppen, Physica E 74, 596 (2015).

[17] H. B. Nielsen and M. Ninomiya, Phys. Lett. B 130, 389 (1983).

[18] M. Büttiker, Phys. Rev. B 46, 12485 (1992).

[19] Ya. M. Blanter and M. Büttiker, Phys. Rep. 336, 1 (2000).

[20] The condition of an adiabatically smooth $h(x)$ is needed for the simplified explanation in Figs. 2 and 4, since it ensures that electrons at all energies are either fully transmitted or fully reflected. We stress that the general results Eqs. (11)-(13) and (16) do not depend on this condition. The magnet transfers the spin by (fully) reflecting the electrons with energies close to the chemical potential (cf. [9]). Since the total spin and charge currents coincide, the charge through the magnet should be carried by the electrons from below the gap independent of the shape of $h(x)$. On the other hand, the electrons with kinetic energy slightly below $-\varepsilon_{\text {gap }}+\hbar \omega / 2$ coming from the left and slightly below $-\varepsilon_{\text {gap }}-\hbar \omega / 2$ coming from the right are always partially reflected. However, these reflections do not affect the spin balance and do not change the total current.

[21] W. Kleeman and F. J. Schäfer, J. Magn. Magn. Mater. 21, 143 (1980).

[22] R. C. O'Handley, Modern Magnetic Materials: Principles and Applications (Wiley, Chichester, UK, 2000).

[23] C. Brüne, A. Roth, H. Buhmann, E. M. Hankiewicz, L. W. Molenkamp, J. Maciejko, X.-L. Qi, and S.-Z. Zhang, Nat. Phys. 8, 485 (2012). 